\title{
Noise from a supersonic round jet discharging into a duct
}

\author{
K.B.M.Q. Zaman ${ }^{1}$ and A. F. Fagan ${ }^{2}$ \\ NASA Glenn Research Center \\ Cleveland, $\mathrm{OH} 44135$
}

\begin{abstract}
In an effort to understand an 'unwanted noise' problem occasionally encountered in ground test facilities, the interaction of a jet flow with a duct is studied in a model scale experiment. While the interaction of subsonic jets was studied earlier, that of supersonic jets is considered in this paper. The effect of the presence of a cylindrical duct in the path of the jet is studied through sound pressure level spectral measurements as well as schlieren flow visualization. When the jet involves screech tones the placement of the duct is found to make only minor effects on the tones themselves as well as on the high frequency noise. However, there is increased energy at low frequencies. The increase in low frequency noise becomes clearer when screech is eliminated from the jet by two small tabs placed at the nozzle exit. It is shown that spectral peaks and increased sound pressure levels occur at frequencies corresponding to the axial acoustic resonance modes of the duct. These peaks persist into the supersonic regime, however, their amplitudes diminish relative to increasing spectral amplitudes at other frequencies with increasing jet Mach number. A wire-mesh screen attached to the end of the duct effectively suppresses such unwanted noise at subsonic as well as supersonic conditions.
\end{abstract}

\section{Introduction}

In large-scale engine tests, a jet engine exhaust is often discharged into a duct or pipe, which in turn is connected to diffusers and other duct sections, to carry the hot flow away and out of the test chamber. In addition to 'regular' jet noise, unwanted high intensity noise is sometimes encountered in such test facilities [1]. In a simplified model scale experiment the unwanted noise could be traced to acoustic resonance modes of the duct excited by the jet turbulence [2]. While resonances of various sections of the entire duct system may come into play, those of the section in the immediate path of the jet are likely to be mostly responsible for the additional noise. When the preferred mode frequency of the jet matches a resonant frequency of the duct there can be a locked-in 'super-resonance' or 'howl'. Even in the absence of a locked-in resonance, high levels of unwanted noise may occur due to the duct modes excited simply by broadband disturbances of the jet. The latter noise will be referred to in the following as 'excited duct mode noise'. The howl is a special case of the excited duct mode noise, and the term 'unwanted noise' is used to cover both.

The unwanted noise is problematic because it can hinder aeroacoustic measurements and also interfere with flow data. It may be difficult or impossible to obtain accurate measurements of the engine's performance characteristics. Worst cases of howl may involve unsteady aerodynamic loads, large enough to raise concerns of structural damage. For example, when such a phenomenon occurred in one of the Arnold Engineering Development Complex's (AEDC) facilities, noise levels of $168 \mathrm{~dB}$ were measured in the vicinity of the test setup [1]. This translates to about $\pm 7 \mathrm{kPa}$ (1 psi) peak-to-peak pressure fluctuation and a casual reader may appreciate the gravity since the corresponding fluctuating force, say, on a $1 \mathrm{mx} 2 \mathrm{~m}$ ( $21 \mathrm{sq} \mathrm{ft})$ door would be about $\pm 21 \mathrm{mPa}$ (3000 lbs).

As stated before, the unwanted noise was investigated earlier in a simplified, model-scale experiment. The results including methods for suppression of the noise, for subsonic jet flows, were summarized in [2]. The experiment involved a small round jet discharging into various cylindrical duct sections. The sharp tone or

\footnotetext{
${ }^{1}$ Inlet \& Nozzle Branch, Aeropropulsion Division, AIAA Associate Fellow.

${ }^{2}$ Optical Instrumentation Branch, Instrumentation and Controls Division, AIAA Associate Fellow.
} 
super-resonance occurred only with ducts of appropriate dimensions (having duct-length-to-nozzle-diameter ratio in specific ranges). In most cases, however, the unwanted noise occurred due to longitudinal resonance modes of the duct excited by jet turbulence. Such excited duct mode noise involved energy concentration in the noise spectra around the resonant frequency with a broad peak rather than a sharp spike, without a sharp and distinct audible tone. Effective suppression of both the super-resonance and the excited duct modes was achieved by wire-mesh screens placed at the ends of the duct. The screen worked better than a number of other methods explored in the experiment. Apparently it worked by dampening the velocity fluctuations at the pressure node and thereby weakening the resonant condition.

Since engine tests often involve supersonic jet exhausts, the model-scale investigation was continued covering the supersonic regime. It is well known that supersonic jets have various noise components [3]. While only turbulent mixing noise occurs with subsonic jets, imperfectly expanded supersonic jets may involve screech tones, broadband shock associated noise, transonic tones, etc. It was thought that the different noise components might interact with the duct acoustic modes to generate a variety of effects. The objective of the continued investigation was to obtain an understanding of those effects and methods for suppression of the unwanted noise.

In view of the vast parameter space, the explorations had to be limited. Only underexpanded jets from a single, round, convergent nozzle were considered. Initially cursory explorations were made with ducts of various sizes. A single cylindrical duct was finally chosen for the detailed study. The duct together with the given nozzle, described further in the next section, approximated the scales of the essential elements of the engine test facility configurations. The screech characteristics for the free jet were first documented. Interactions of specific screech modes with the duct were examined in a systematic manner. Using two 'tabs', screech was then eliminated allowing an investigation of the interaction of screech-free supersonic jets with the duct. The effect of wire-mesh screens for suppression of the unwanted noise was also explored. The results, based on sound pressure level measurements at a fixed location and schlieren flow visualization, are discussed in the following.

\section{Experimental Facility and Procedure}

The experiments were conducted in a small open jet facility at NASA Glenn Research Center (GRC). Compressed air passed through a plenum chamber and then exhausted through a convergent nozzle of diameter, $d=1.47 \mathrm{~cm}$ (0.58 in); a picture of the setup is shown in Fig. 1(a). The duct, a straight cylindrical pipe section, was placed in the jet's path aligned with its axis. The duct's dimensions (diameter x length) were $5.08 \times 12.7 \mathrm{~cm}(2 \times 5 \mathrm{in})$. It was mounted on a 3-axis traversing mechanism that enabled manual positioning in lateral directions and computer controlled positioning in the streamwise direction. The standoff distance from the exit of the nozzle to the entrance of the duct is denoted by $s$.

Sound pressure level measurements were made with a $1 / 4$-inch microphone (B\&K 4135) held fixed at a distance 53d and approximately at $95^{\circ}$ relative to the jet's downstream axis. A PC-based data system with 'Labview' software was used for all data acquisition and analysis. Most spectral data were acquired over 0-50kHz range with 800-line analysis (bandwidth of $62.5 \mathrm{~Hz}$ ). A few sets were analyzed over $0-10 \mathrm{kHz}$ with $25 \mathrm{~Hz}$ bandwidth resolution in order to focus on the low frequency content.

The effect of a wire-mesh screen (70 mesh per inch having 30\% openness ratio) placed at the downstream end of the duct was explored for noise suppression. Pictures of the duct with and without the screen are shown in Fig. 1(b). Two tabs were used on the nozzle for suppression of screech tones, so that the interaction of screech-free supersonic jets with the duct could be studied. The total area blockage by the two triangular shaped tabs was slightly less than $2 \%$ of the nozzle's exit area. A picture of the nozzle with the tabs is shown in Fig. 1(c). All noise spectral data are presented as measured without any correction or normalization. 
A focusing schlieren system was used for flow visualization. The light source illuminating the source grid was a 5 Watt Xenon flashlamp with 1 micro-second pulse duration. All optical components as well as the scientific-grade CCD camera were securely housed in a 58x43x25 cm case. The case was placed on one side of the jet while the $76 \times 61 \mathrm{~cm}$ retro-reflective screen was placed on the other side. The distances of the two items from the jet's center plane dictated the size of the field of view. The chosen distances, within the constraints of the test chamber, provided a field of view that extended approximately $24 \mathrm{~cm}$ in the streamwise direction. The thickness of the focused field was estimated to be about $7.5 \mathrm{~cm}$. The pictures shown in the following are essentially instantaneous snapshots of the density gradients in the flowfield capturing the shocks and vortical structures of the flow. Further details of the schlieren system can be found in [4].

\section{Results}

The screech tone frequencies were measured by spectral analysis of the microphone signal. The bandwidth for these measurements was $25 \mathrm{~Hz}$ which essentially determined the uncertainty of the frequency data $( \pm 12.5 \mathrm{~Hz})$. The 'jet Mach number' $\left(M_{j}\right)$ was determined via isentropic equations from the ratio of the plenum and ambient pressures (i.e., Mach number had the flow expanded fully). The plenum pressure was held within $3.45 \mathrm{kPa}(0.5 \mathrm{psi})$ of the set value; this provided an accuracy of $M_{j}$ within $2 \%$ at the low end of the range covered, the accuracy being better at higher values of $M_{j}$. Figure 2 shows screech frequency variation with $M_{j}$. As it is well known [5, 6], the frequency variation goes through various stages with jumps to lower or higher values involving some overlaps (hysteresis). By comparing with screech stages reported in the literature differences are noted. There are additional jumps within the given $M_{j}$-range. Following the nomenclature for screech stages in the literature, the observed stages are denoted as shown in the legend. The main difference with data in the literature is that stages $B$ and $C$ appear to go through additional jumps. Based on past reports, stages A1 and A2 are conjectured to involve axisymmetric, stages B and D flapping, and stages $C$ and $E$ helical unsteady perturbation in the jet. For the purposes of the present study, the conditions at four values of $M_{j}$, representing the $\mathrm{A} 1, \mathrm{~B}, \mathrm{~B}+\mathrm{C}$ and $\mathrm{D}$ stages are chosen for studying their interaction with the duct. The four $M_{j}$, with nominal values of 1.11, 1.27, 1.38 and 1.60, are marked by the vertical dashed lines in Fig. 2.

Figure 3 compares sound pressure level (SPL) spectra for the free jet (no tabs) with the case of the duct (no screens) placed in the jet's path, at the four values of $M_{j}$. The duct stand-off distance $s$, noted in the figure caption, is normalized by $d$. Data for the lowest $M_{j}$ are shown in Fig. 3(a); the solid (red) line represents the free jet while the dashed (blue) line is for the case with the duct. In the legend the actual value of $M_{j}$ followed by overall sound pressure level (OASPL) in $\mathrm{dB}$ are listed; notation 'FJ' stands for free jet and '2x5-NS' stands for the 2x5-in. duct open on both ends (no screen). The screech component (at $15.1 \mathrm{kHz}$ ) has changed slightly while the spectral shape at low frequencies has changed drastically. There is a peak around $1.1 \mathrm{kHz}$ that approximately corresponds to the half-wave longitudinal resonance of the $12.7 \mathrm{~cm}$ long duct (with end corrections taken into account [2]). The peak (shown with better resolution in a later figure) is not as sharp as the spike at $15.1 \mathrm{kHz}$ and represents the excited duct mode noise defined in $\S 1$. Note that the OASPL has increased in this case with the presence of the duct from $110.5 \mathrm{~dB}$ to $113.9 \mathrm{~dB}$. At higher $M_{j}$ the effect of the duct is similar except that OASPL has actually decreased somewhat and the excited duct mode noise is more prominent at the second harmonic (around $3.3 \mathrm{kHz}$ ). At all $M_{j}$ the screech fundamental frequency is seen to shift to a slightly lower frequency when the duct is present. The shift could have occurred due to a change in the screech feedback loop caused by the presence of the duct. The data trends are further discussed in the following.

The effect of duct standoff distance $s$ is examined in Fig. 4. The four figures in (a)-(d) are for the four values of $M_{j}$. In each figure, the spectral traces are for different $s$ as marked; (the ordinate scale pertains to the trace at the bottom while the others are staggered by 1 division). At the lowest $M_{j}$ in (a), screech amplitude is seen to diminish for low values of $s$. However, at higher values of $s$ screech is again prominent. The presence of the duct has reduced the screech frequency for $s$ values up to 1.6. At $M_{j}=1.27$ in (b), screech frequency has increased at first and then decreased with increasing $s$. A similar trend is noted at $M_{j}=1.38$, however, closely-spaced multiple peaks are noted at small values of $s$. The overall trend in frequency variation with $s$ is seen to change again at the highest $M_{j}$ covered 
in the experiment. It is clear, however, that for large $s$ screech returns to the same frequency as with the free jet, at all $M_{j}$. The screech frequencies are 15.1, 9.3, 8.3 and $6.7 \mathrm{kHz}$ at $M_{j}=1.11,1.27,1.38$ and 1.60, respectively.

Schlieren pictures of the free jet at the four values of $M_{j}$ are shown in Figs. 5(a)-(d). The shock structures are clearly seen. The shock spacing progressively increases with increasing $M_{j}$. Upon a closer inspection, radiating acoustic waves can be noticed in most of the pictures. However, the unsteady vortical motion (whether axisymmetric, flapping or helical) cannot be discerned from these pictures. Pictures for the duct case, corresponding to the same $M_{j}$ 's of Fig. 5, are shown in Fig. 6. Comparing the two sets of pictures, it is difficult to discern any differences in the flow upstream of the duct. Some changes in the shock structure are visible, e.g., at the lowest $M_{j}$. Downstream of the duct the jet appears to be larger in diameter, relative to the free jet case, especially at the higher values of $M_{j}$. Figure 7 shows schlieren pictures for four values of $s$, all for $M_{j}=1.38$. Referring back to Fig. 4(c), it is seen that the screech frequency has reduced somewhat for duct positions $s=1.6$ or $s=3.2$ ( $7.6 \mathrm{kHz}$ compared to $8.3 \mathrm{kHz}$ for the free jet). An interrogation of the pixel counts in the picture suggests that the shock-spacing, seen upstream of the duct at $s=1.6$ and 3.2 (Figs. 7c and 7d), has not changed relative to the free jet case (Fig. 5c). The average spacing for the first two shocks is determined to be $1.15 d$ in all three cases.

Thus, the shock-spacing has remained constant while there is a change in screech frequency. This may appear contradictory but is possible through a stage jump or an influence of the duct on the feedback path. While within a stage a decrease in screech frequency corresponds to an increase in shock-spacing, a stage jump at a given $M_{j}$ does not involve a noticeable change in the shock-spacing [7]. Furthermore, as stated, it is possible that the presence of the duct has altered the screech feedback loop causing a change in the frequency. In any case, the data in Figs. 3-7 indicate that the overall effect of the duct on the screech tones and the shock-structure of the jet are rather minimal. However, low frequency noise is generated due to the excited duct modes, and this is further examined in the following with the screech-free jet obtained by using the tabs.

First, the effect of the tabs on free jet noise is documented in Fig. 8. Pairs of SPL spectra are shown, with and without the tabs, for the four values of $M_{j}$ in a similar manner as with Fig.3; the notation ' $F J t$ ' in the legend stands for free jet with the tabs (Fig. 1c). Note the large change in the spectral shape at all four $M_{j}$. The screech tone(s) are gone and the OASPL has decreased substantially in all cases. The elimination of screech has unearthed the broadband shock-associated noise (BBSN) [3, 8], represented by the broad peaks in the spectra clearly visible at the higher values of $M_{j}$. The frequency of the BBSN peak drops with increasing $M_{j}$; the peaks are centered around 33, 25 and $18 \mathrm{kHz}$ for $M_{j}=1.27,1.38$ and 1.60 , respectively.

The effect of the duct for the tabbed jet is shown in Fig. 9. SPL spectra at several $M_{j}$ are shown over the range 0$10 \mathrm{kHz}$ ( $25 \mathrm{~Hz}$ bandwidth analysis). In addition to the supersonic cases, spectral traces at several subsonic conditions are also shown for comparison. The outstanding feature of this plot is the fact that the duct resonance mode at about $1.1 \mathrm{kHz}$ is excited at all conditions - subsonic or supersonic. This is emphasized by the vertical dashed line drawn in the figure. As stated before, this is due to the half-wave longitudinal resonance ( $m=0$ mode) of the duct and represents the excited duct mode noise. It is also obvious that the broadband noise of the jet grows in amplitude as $M_{j}$ is increased. This tends to drown out the $1.1 \mathrm{kHz}$ spectral peak at high values of $M_{j}$. Note also that in all cases there is energy concentration around $3.3 \mathrm{kHz}$ which is the first harmonic of longitudinal acoustic resonance for the duct open on both ends. Furthermore, there might be energy due to excitation of other resonance modes ( $m=1, \pm 1$, etc) but none of those are clear. In the present as well as the earlier study [2], the longitudinal resonance modes are found to be dominant contributing mostly to the unwanted noise.

The effect of the duct, with and without a 70-mesh screen on the end, is shown in Fig. 10 with SPL spectra over 0$50 \mathrm{kHz}$ range. First compare the green solid curves (free tabbed jet) with the blue dashed curves (duct having no screen). It is noted that the BBSN peak has been drastically reduced by the presence of the duct. BBSN can be explained by constructive reinforcement of noise radiated from multiple sources, at the observation location, the shocks acting as the sources [8]. Thus, with the duct in the path the radiated noise from some of the sources are blocked and this may explain the observed effect. It is noted that there is increased energy at low frequencies for the 
duct case (blue dashed line), as also seen in Fig. 3 for the case without the tabs. For reasons not clearly understood, the screech tone has reappeared in Fig. 10(c) at $M_{j}=1.38$ for the tabbed jet with the presence of the duct.

The use of the screen (red solid lines in Fig. 10) has made only small differences at high frequencies (compare with the blue dashed line). However, there is a significant effect on the excited duct mode noise at low frequencies. This is shown clearly in Fig. 11 where data with and without the screen are compared over an abscissa range of $0-10 \mathrm{kHz}$ ( $25 \mathrm{~Hz}$ bandwidth). Also included in this figure is a set of data at subsonic condition of $M_{j}=0.715$. (In order to maintain correspondence with the notations (a)-(d) of other figures the latter data is shown out of sequence at the bottom in Fig. 11e). In all figures, data for the tabbed jet are compared with and without the screen on the duct. In all no-screen cases, the excited duct mode spectral peak around $1.1 \mathrm{kHz}$ is discernible which becomes more conspicuous as $M_{j}$ is decreased (as also seen in Fig. 9). It is noted that the amplitude of this peak rises from $91 \mathrm{~dB}$ to about $96 \mathrm{~dB}$ as $M_{j}$ is increased from 0.715 to 1.11 but then it remains a constant in the supersonic regime. The screen suppresses this peak. The effect appears more pronounced at lower values of $M_{j}$. where the peak is more prominent to begin with. It is also noteworthy that the screech component that reappeared for $M_{j}=1.38$ (around 9.7 kHz; Figs. 10c and 11c), has also been suppressed by the screen.

Schlieren pictures for the tabbed jet with and without the screen on the duct are presented in Figs. 12, 13, 14 and 15 for $M_{j}=1.11,1.27,1.38$ and 1.60, respectively. In each figure the free tabbed jet data are shown at the top followed by the duct case in the middle and the duct with screen case at the bottom. First, by comparing the pictures on the top with the no-tab free jet data (Fig. 5) it can be seen that the tabs have drastically altered the shock structure in the jet. The shock structure is no longer well defined and the shock-spacing has reduced. By comparing the top and middle pictures for each figure, it is evident that the presence of the duct has not made a significant impact on the shock structure upstream. The same can be said for the duct with the screen (picture at bottom). Recall that a similar inference was made for the jet without tabs. The impact of the screen on the flow downstream of the duct, however, is clear. The flow is clearly more mixed exhibiting a less granular structure. This is true at all four values of $M_{j}$ in Figs. 12-15.

\section{Concluding Remarks}

An investigation is carried out on noise from the interaction of supersonic jets with a duct. The study is a continuation of an earlier work [2] that addressed such interaction for subsonic jets. Underexpanded supersonic jets, from a round convergent nozzle, up to jet Mach numbers of 1.63 are covered in this study. The interaction with a single cylindrical duct is explored; the nozzle and duct dimensions are such as to approximate typical scales of practical engine test facilities. Results are first obtained with natural screech tones present with the jet, and then with screech-free jets, the screech having been eliminated by the use of two small tabs at the nozzle exit. It is inferred that the excited duct mode noise, i.e., acoustic resonance modes of the duct excited by the jet turbulence (without a locked-in super-resonance), persists well into the supersonic regime albeit with diminishing relative amplitude with increasing $M_{j}$. With increasing $M_{j}$, the amplitude of this spectral peak is seen to grow in the subsonic regime but becomes a constant in the supersonic regime. Thus, since the spectral amplitudes at other frequencies continue to grow with increasing $M_{j}$, the excited duct mode spectral peak gradually drowns out. However, it is still detectable at the highest $M_{j}$ covered in the experiment. A wire-mesh screen is found to effectively suppress the excited duct mode spectral peak, for subsonic as well as supersonic jets. It should be noted that with the given nozzle and duct configuration a super-resonance or howl was not encountered. However, it was shown in [2] that with certain combinations of geometries super-resonance occurred readily and the screen was also effective in completely suppressing it.

The fact that the excited duct mode noise can be suppressed throughout the jet Mach number range, especially at high subsonic and transonic jet Mach numbers, is significant from an application point of view. It is thought that much of the unwanted noise in test facilities is due to the excited duct modes and not necessarily due to superresonance. It is also noteworthy that various methods have been proposed previously for noise reduction in engine test stands, especially in several US patents (L.R. Bridge, 2823756, 1958; M. Hirschorn, 2864455, 1958; J.M. Tyler 2936846, 1960; B.D. Blackwell, 2979151, 1961; G.M. Lilley, 2987136, 1961; M.L. Morris, 
3120877, 1964; W.B. Shearer, 3159238, 1964; E.H. Miller, 3165167, 1965; C.D. Smith, 3187835, 1965; R.A. Putnam, 7717229 B2, 2010; and N.L. Helgeson, 7918310 B1, 2011). All of these patents, except the one in Ref. [9], dealt with noise reduction in cases where a high velocity flow would otherwise be exhausted directly into the atmosphere causing the high levels of noise. The concepts basically involved fragmenting the exhaust flow and thereby reducing its velocity before discharging into the atmosphere. The reduced velocity, together with acoustic linings, would yield the noise attenuation. The present result is distinct in that it pertains to test facilities where flow conditioners are used so that the final exhaust velocity is already low, enough not to produce significant noise pollution. Yet, as discussed in §1, these facilities occasionally produce the unwanted noise due to resonance. The present results suggest a method for alleviating the problem.

The previous work of K. W. Kinzie [9], had also addressed the resonance in engine test facilities and offered a method for suppressing the howl. The method involved insertion of a rod perpendicular to the flow. This was based on experimental observation and apparently worked due to a disruption of the feedback loop of the resonance, the exact process was not clearly understood. The method required trial and error for finding the optimum rod position for each operating condition. Results reported in [2] also showed that while the rod may have suppressed the howl it was ineffective in suppressing the excited duct mode noise. The current results suggest a possible permanent fix of the problem by positioning a wire-mesh-like structure at the end of the duct section suspected of being responsible for the resonance. This assumes that the dominant contributors for the unwanted noise are the duct's longitudinal acoustic resonance mode and its odd harmonics. It is suspected that is indeed the case for the unwanted noise in many facilities observed in the past. A screen-like structure apparently works by dampening the velocity perturbation at the pressure node (velocity anti-node) and may suppress not only the howl but also the excited duct mode spectral peaks. Furthermore, the technique appears to be most effective at high-subsonic to low supersonic conditions, which apparently is the flow regime where most of the unwanted noise and howl were encountered in the past.

\section{Acknowledgement}

Support from the Aeronautical Sciences Project of NASA's Fundamental Aeronautics Program is gratefully acknowledged.

\section{References}

[1] Jones, R.R. and Lazalier, G.R., "The acoustic response of altitude test facility exhaust systems to axisymmetric and two-dimensional turbine engine exhaust plumes”, DGLR/AIAA Aeroacoustics Conference, Aachen, Germany, AIAA Paper 92-02-131, May, 1992.

[2] Zaman, K.B.M.Q., Clem, M.M. and Fagan, A.F., "Noise from a jet discharged into a duct and its suppression”, Int. Journal of Aeroacoustics, vol. 12, no. 3, pp. 189-214, 2013

[3] Tam, C.K.W., “Supersonic jet noise,” Ann. Review Fluid Mech., 27, pp. 17-43, 1995.

[4] Fagan, A.F., L’Esperance, D. and Zaman, K.B.M.Q., “Application of a novel projection focusing schlieren system in NASA test facilities”, AIAA Paper 2014-xxxx, Aviation and Aeronautics Forum and Exposition, Atlanta, June 14-20, 2014.

[5] Raman, G., "Advances in understanding supersonic jet screech: review and perspective”, Progress in aerospace Sciences 34, no 45, p. 106 (also, AIAA Paper 98-0279), 1998.

[6] Norum, T. D., "Screech suppression in supersonic jet”, AIAA J. 21, 235-240, 1983.

[7] Clem, M. M., Zaman, K.B.M.Q. and Fagan, A.F., "Background oriented schlieren applied to study shock spacing in a screeching circular jet”, AIAA Paper 2012-0403, 50 ${ }^{\text {th }}$ Aerospace Sciences Meeting, January 912, 2012, Nashville, TN.

[8] Harper-Bourne, M., and Fisher, M. J., “The Noise from ShockWaves inSupersonic Jets,” Proceedings of the AGARD Conference on Noise Mechanisms, AGARD CP 131, 1973.

[9] Kinzie, K.W., "Methods and apparatus for suppressing engine test cell howl”, US patent 6092621, 2000. 


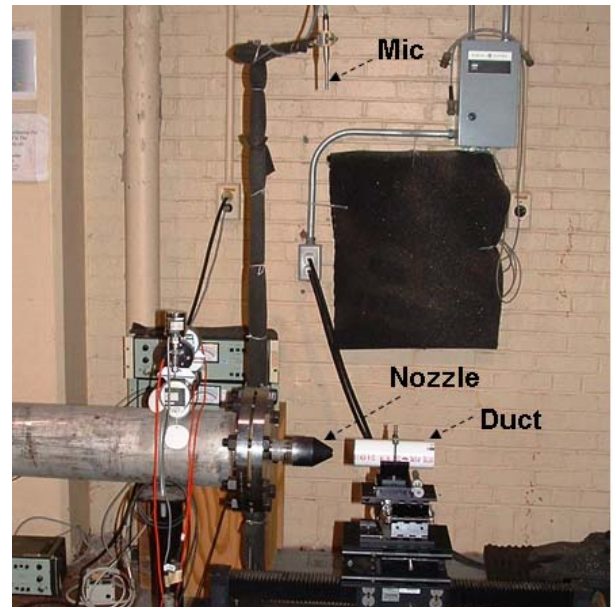

(a)

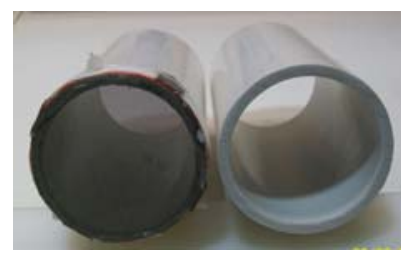

(b)

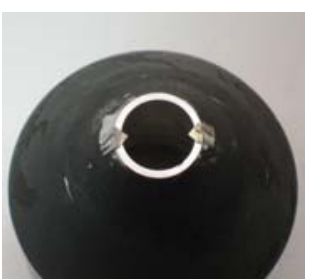

(c)
Fig. 1 Experimental facility. (a) Overall set-up, (b) 2 "x5" ducts, one on left has 70-mesh screen on end, (c) nozzle fitted with 2 tabs.

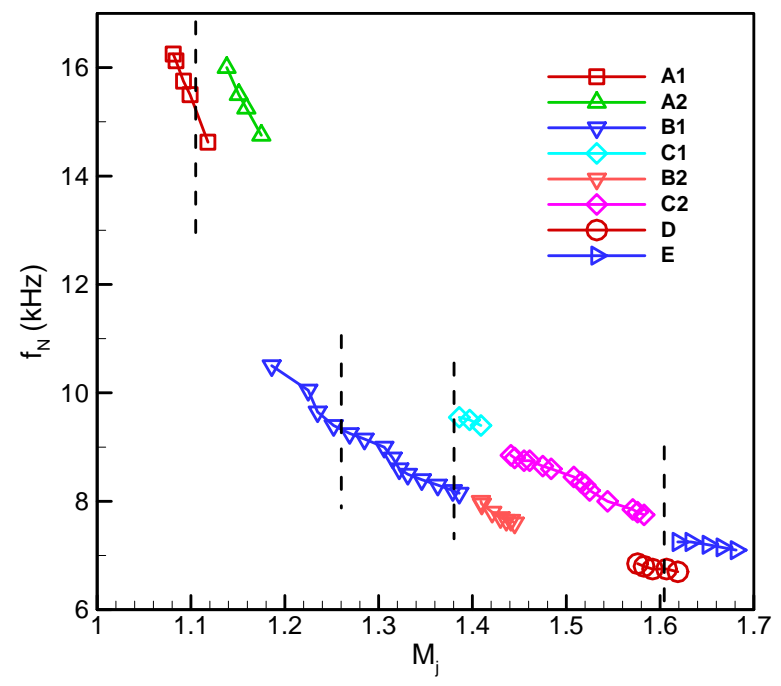

Fig. 2 Screech frequency versus $M_{j}$ for the $d=0.58$ in. jet; screech 'stages' are identified in the legend.

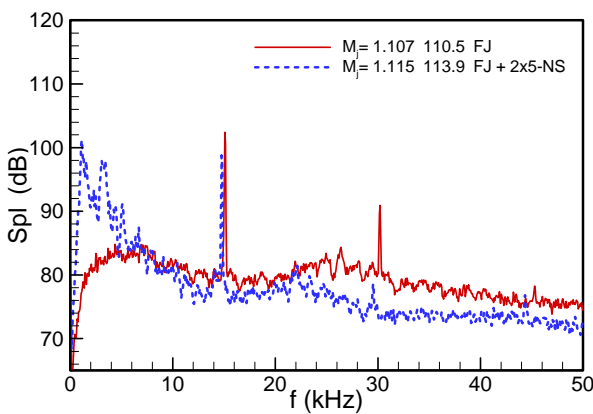

(b)
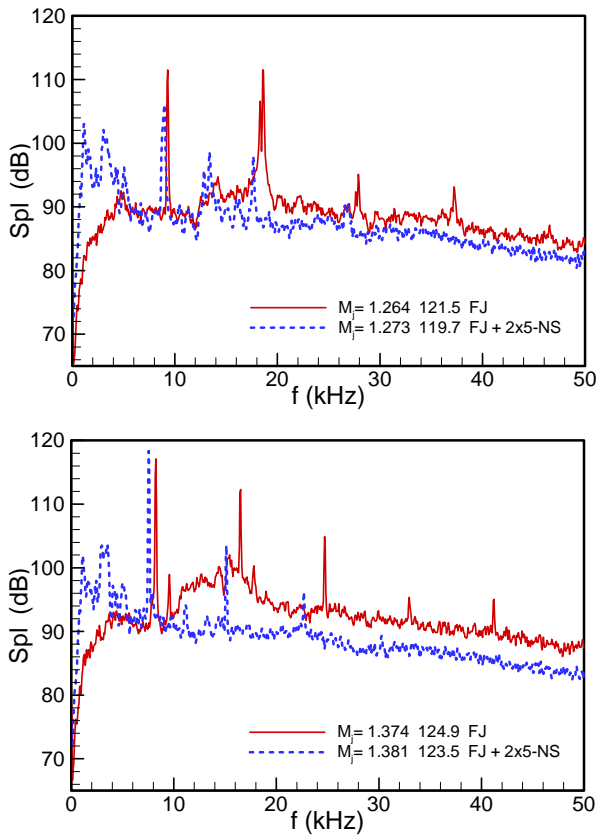

(d)

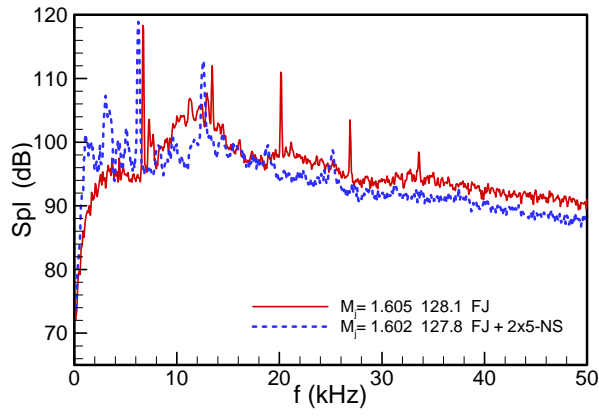

Fig. 3 SPL spectra. Red (solid) line: free jet, Blue (dotted) line: with the duct in the jet's path $(s=3)$.

(a) $M_{j}=1.11$, (b) $M_{j}=1.27$, (c) $M_{j}=1.38$, (d) $M_{j}$ $=1.60$. The second number in the legend represents OASPL in $\mathrm{dB}$. 
(a)
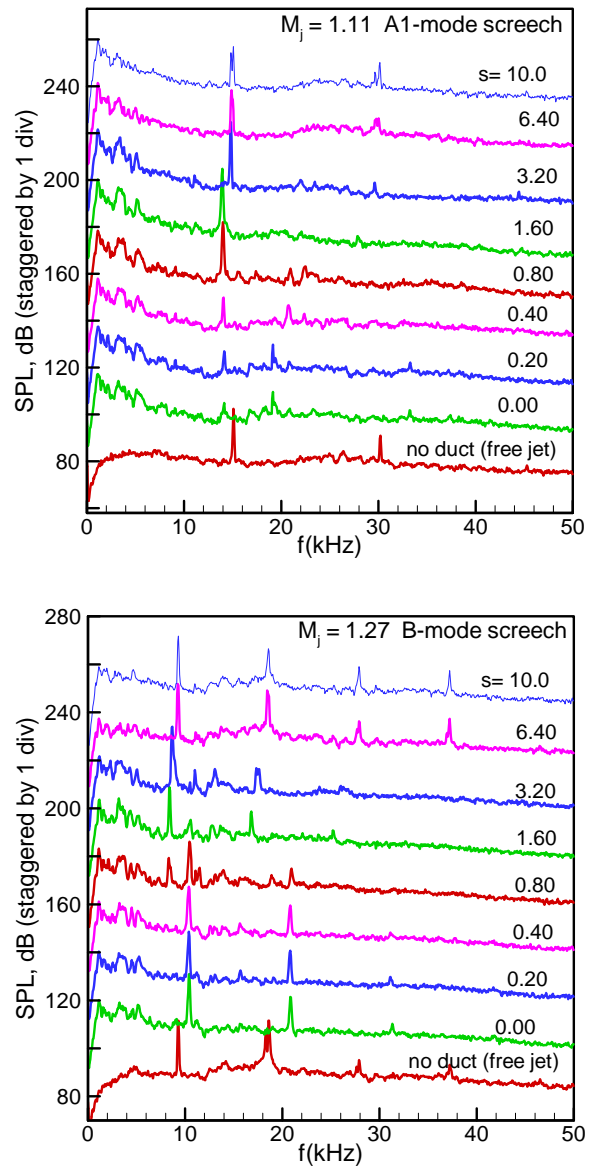

(b)

Fig. 4 Caption next column

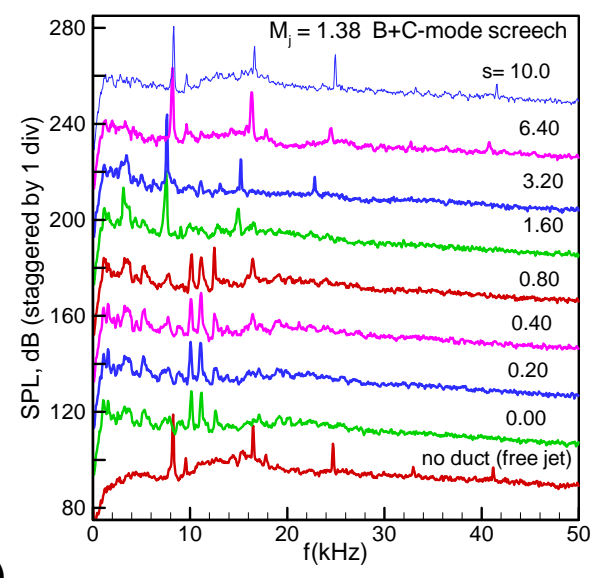

(c)

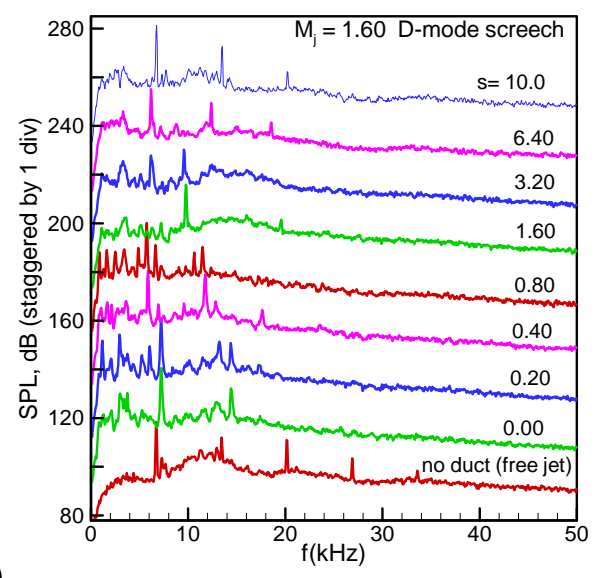

(d)

Fig. 4 Composite plots of SPL spectra for varying standoff distance (s) of the duct. (a) $M_{j}=1.11$, (b) $M_{j}=1.27$, (c) $M_{j}=1.38$, (d) $M_{j}=1.60$. 


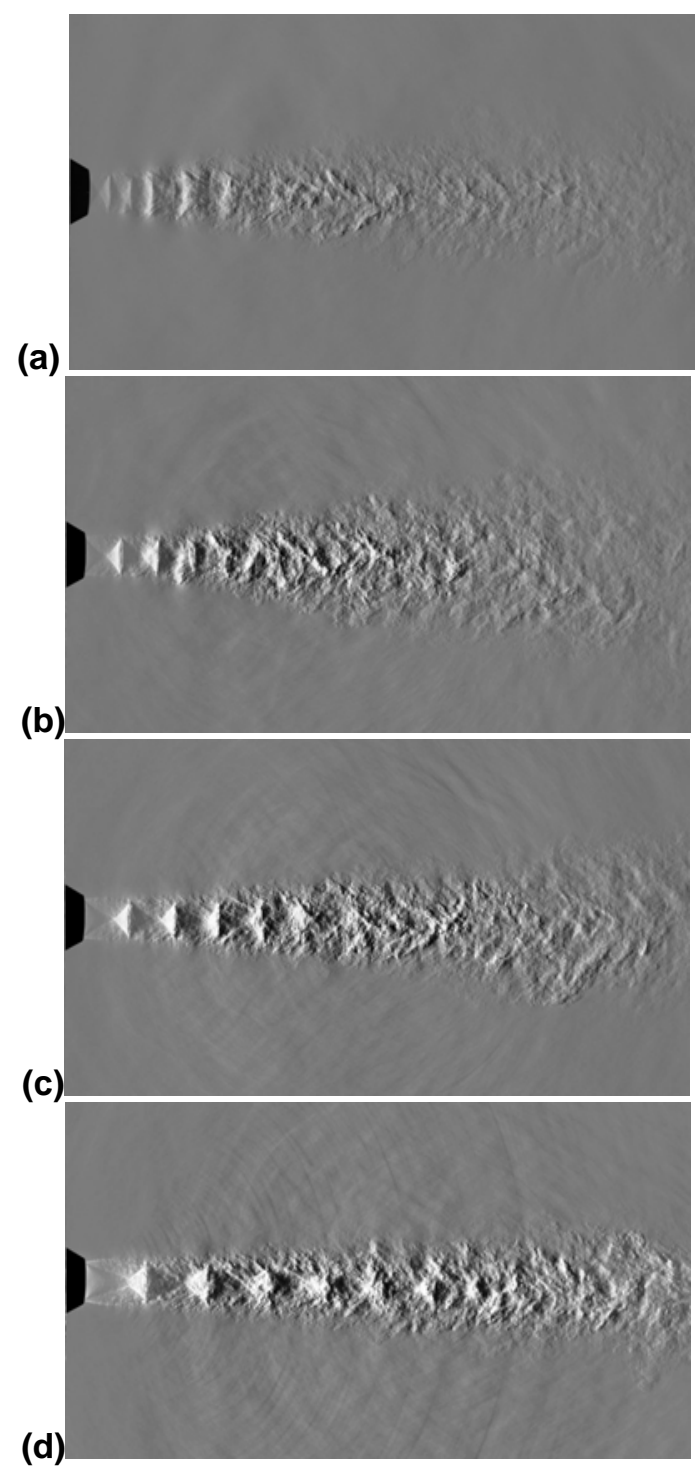

Fig. 5 Schlieren pictures of the free jet; (a) $M_{j}$ =1.11, (b) $M_{j}=1.27$, (c) $M_{j}=1.38$, (d) $M_{j}=1.60$.

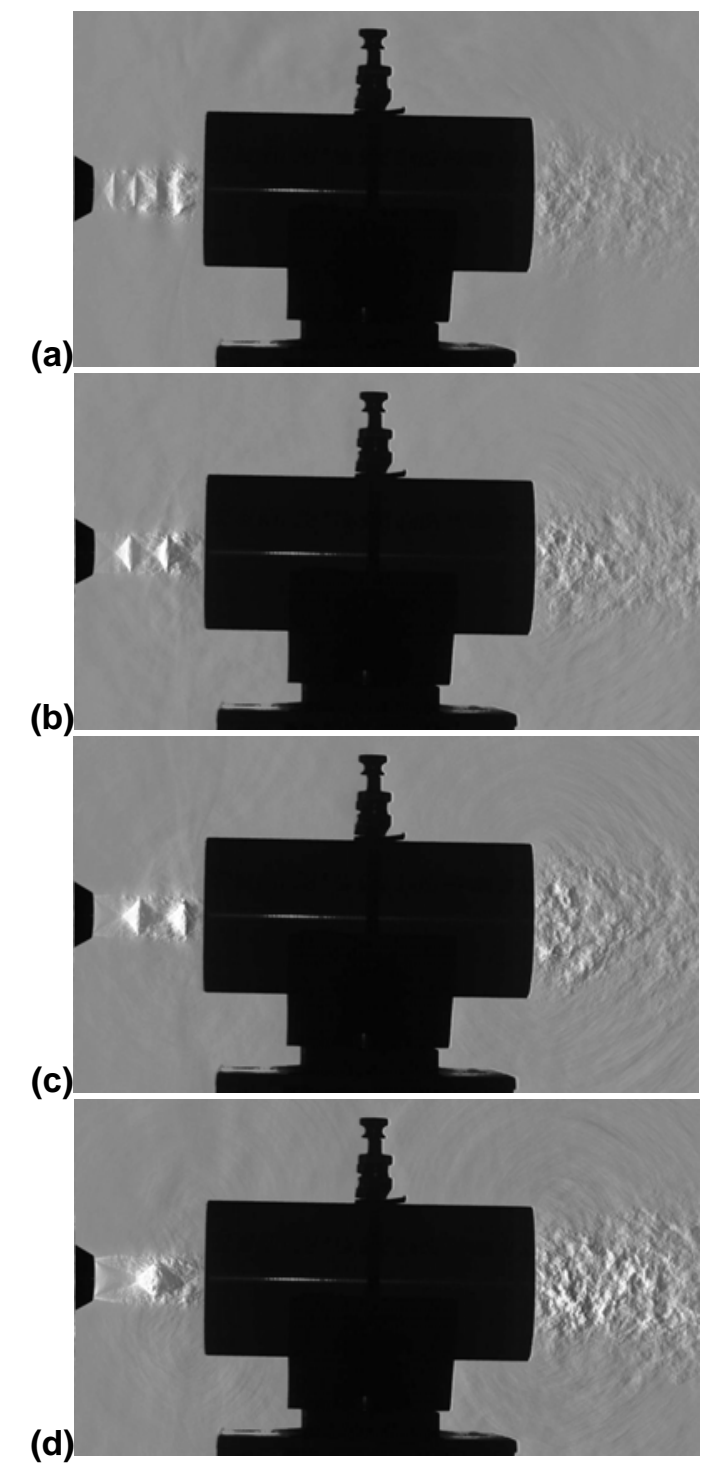

Fig. 6 Schlieren pictures of jet discharging into the duct at s=3; (a) $M_{j}=1.11$, (b) $M_{j}=1.27$, (c) $M_{j}$ $=1.38$, (d) $M_{j}=1.60$. 


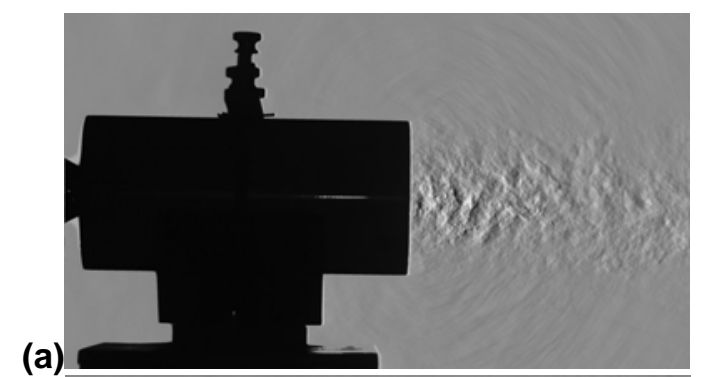

\section{(a)}

(b)

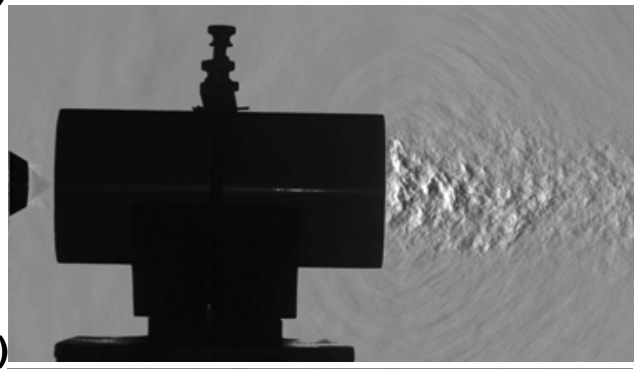

(c)

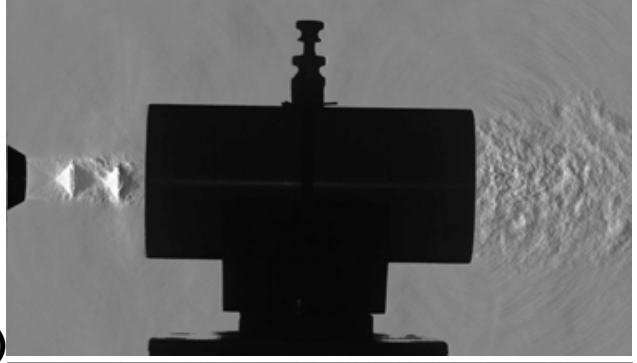

(d)

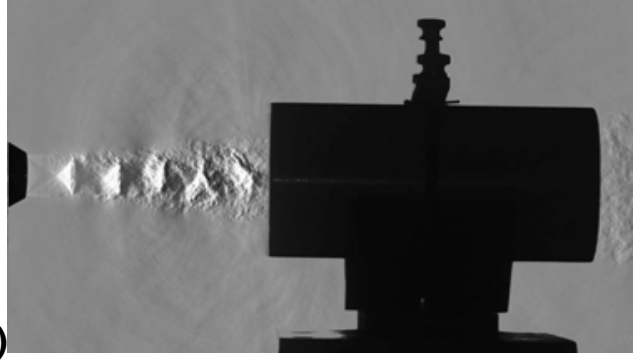

Fig. 7 Schlieren pictures of jet at $M_{j}=1.38$ discharging into the duct with varying $s$; (a) $s=0$, (b) $s=0.8$, (c) $s=1.6$, (d) $s=3.2$.

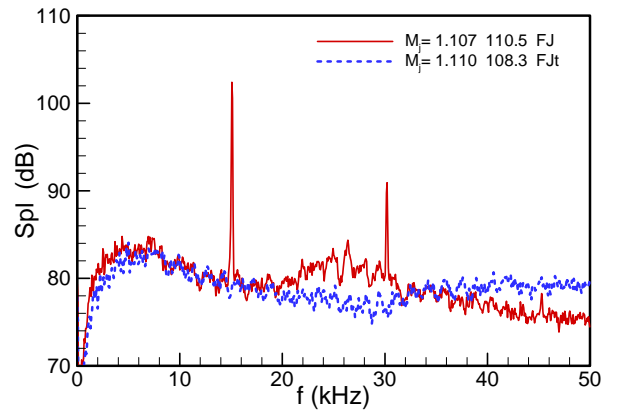

(a)

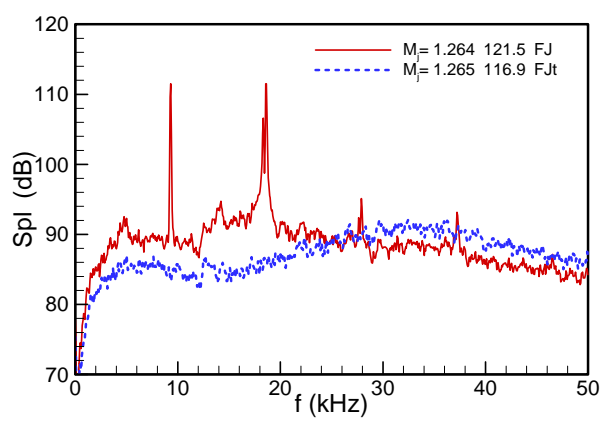

(c)

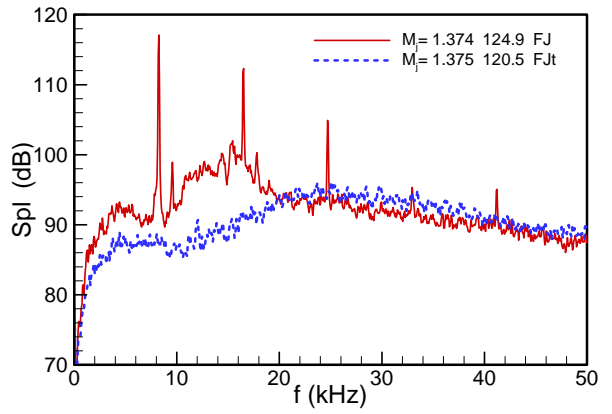

(d)

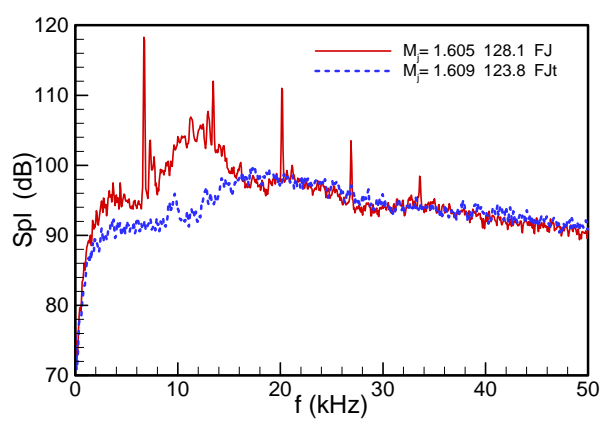

Fig. 8 SPL spectra for free jet. Red (solid) line: normal nozzle, Blue (dotted) line: nozzle with 2tabs. (a) $M_{j}=1.11$, (b) $M_{j}=1.27$, (c) $M_{j}=1.38$, (d) $M_{j}=1.60$. 


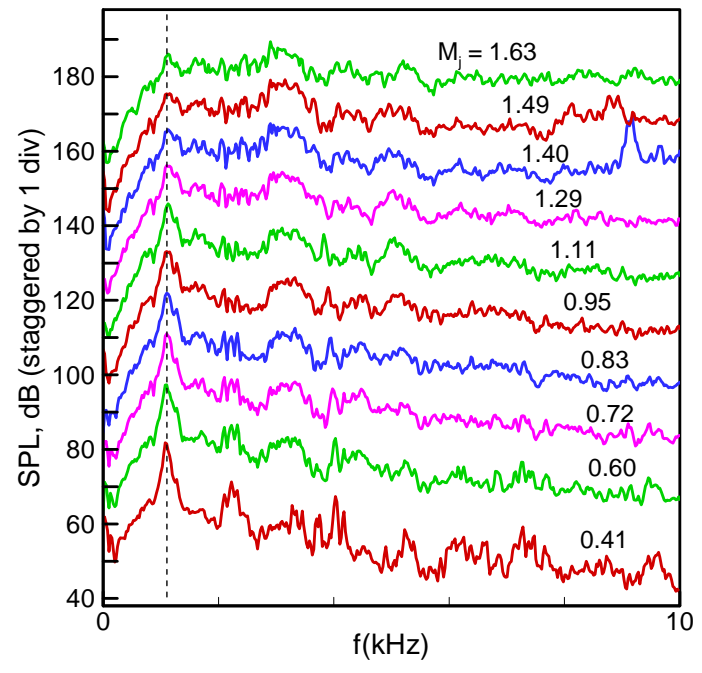

Fig. 9 SPL spectra for the tabbed jet discharging into the duct at $s=3$, for different jet Mach number $M_{j}$ as indicated. (a)
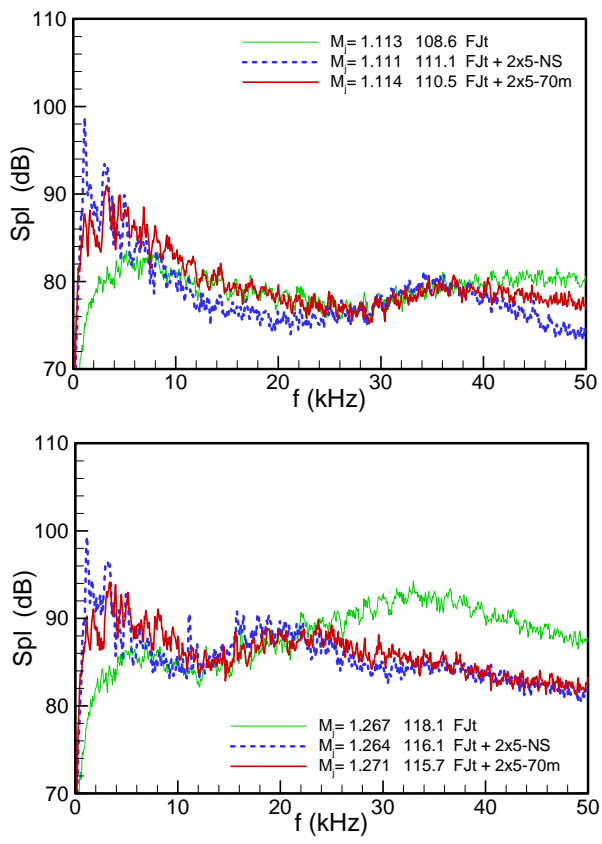

(b)

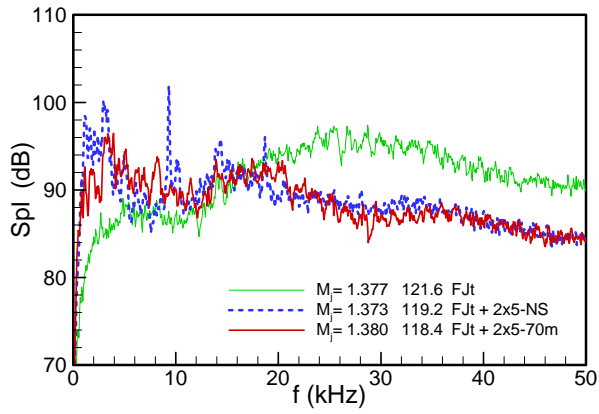

(c)

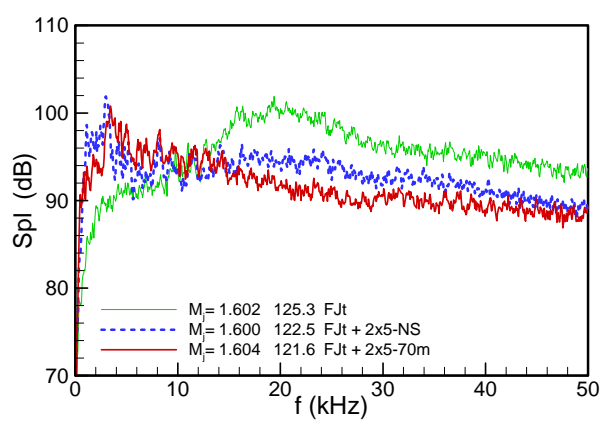

Fig. 10 SPL spectra for the tabbed jet with and without the duct ( $s=3)$; green (solid) line: free jet, blue (dashed): duct with no screen, red (solid): duct with $70 \mathrm{~m}$ screen on downstream end. (a) $M_{j}$ =1.11, (b) $M_{j}=1.27$, (c) $M_{j}=1.38$, (d) $M_{j}=1.60$. 
(a)

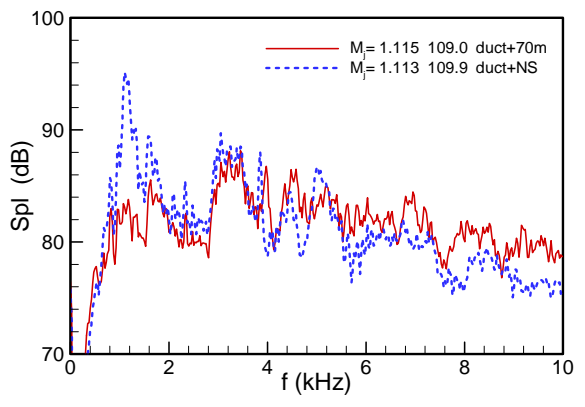

(b)

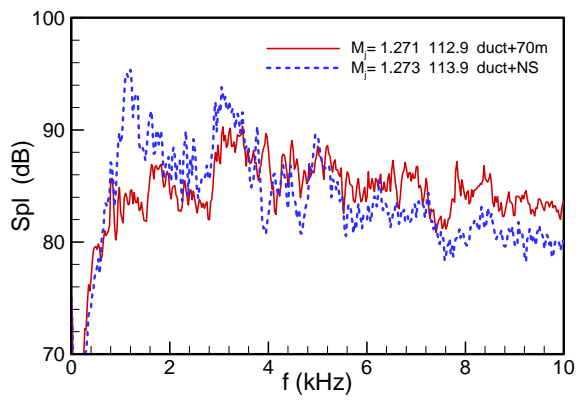

(c)

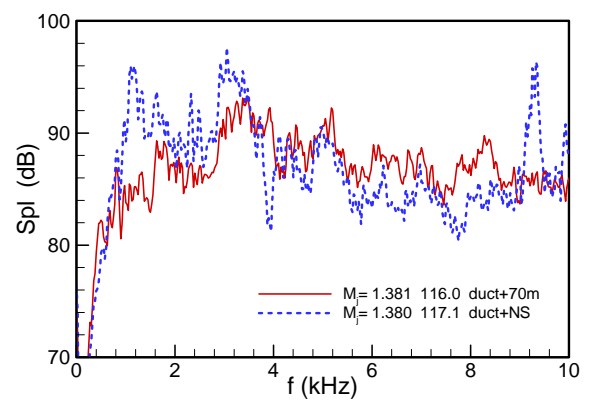

(d)

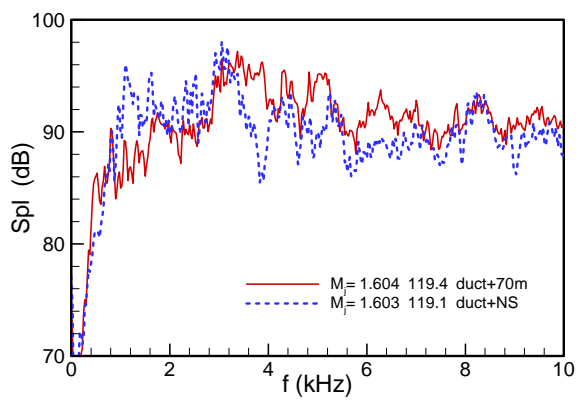

(e)

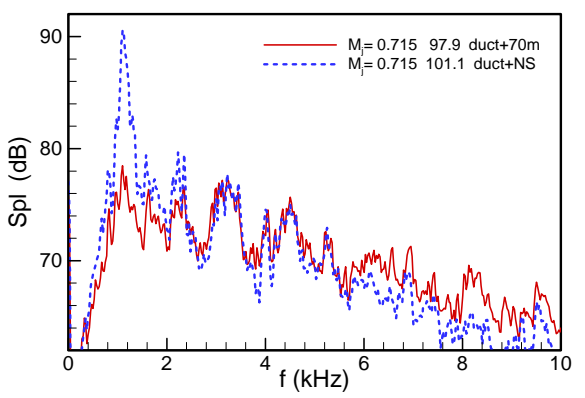

Fig. 11 SPL spectra for tabbed jet plus duct with and without screen ( $s=3$ ); blue (dashed): no screen, red (solid): $70 \mathrm{~m}$ screen on downstream end of duct. (a) $M_{j}=1.11$, (b) $M_{j}=1.27$, (c) $M_{j}$ =1.38, (d) $M_{j}=1.60$, (e) $M_{j}=0.71$. (a)

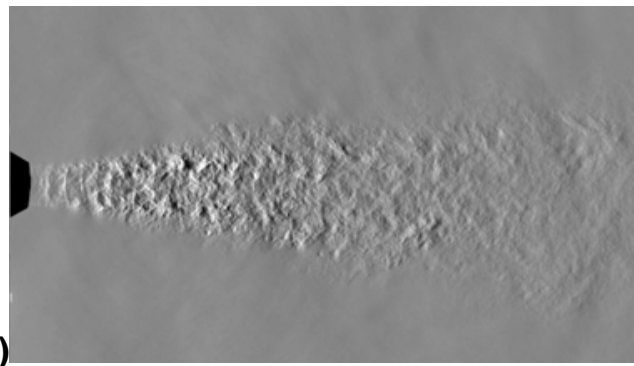

(b)

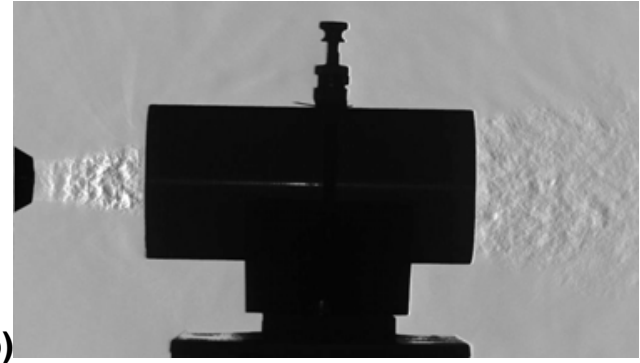

(c)

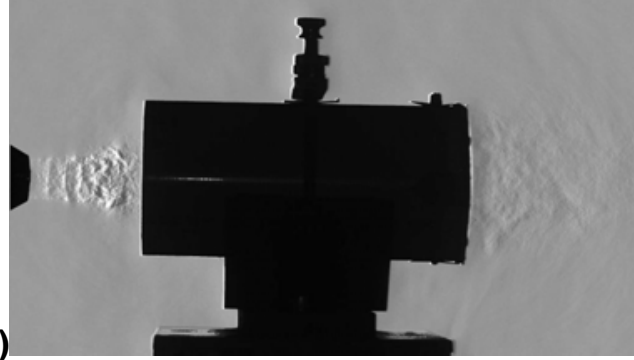

Fig. 12 Schlieren pictures. (a) free tabbed jet, (b) tabbed jet plus duct ( $s=3$ ), (c) tabbed jet plus duct with 70-mesh screen at end ( $s=3) ; M_{j}=1.11$. 
(a)
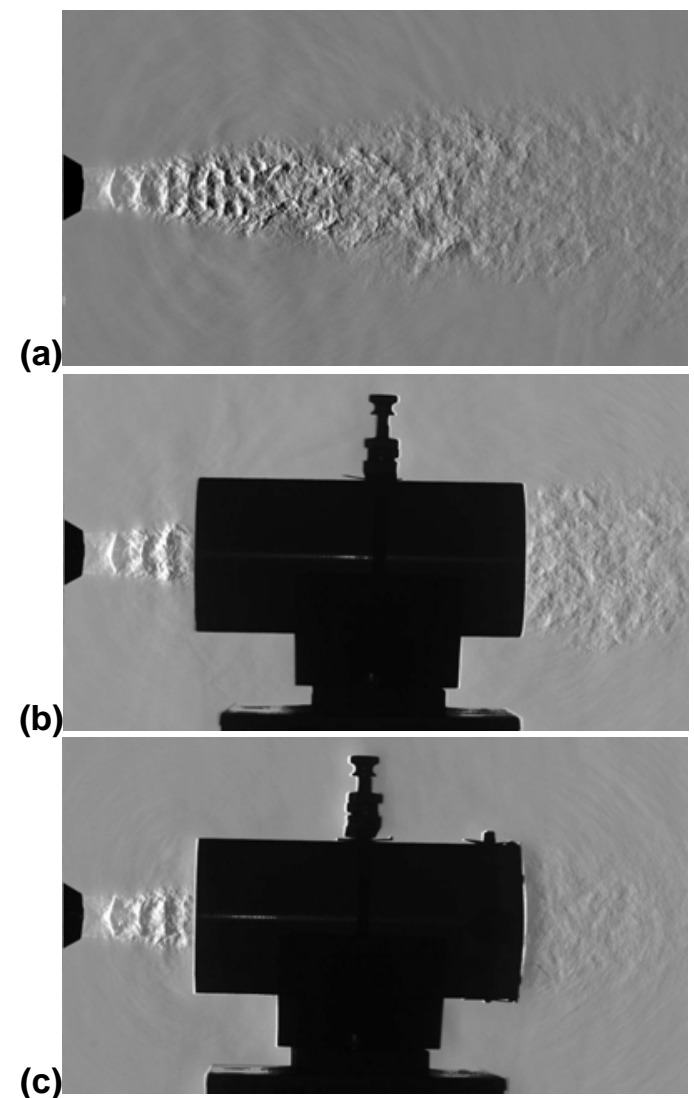

Fig. 13 Schlieren pictures. (a) free tabbed jet, (b) tabbed jet plus duct ( $s=3$ ), (c) tabbed jet plus duct with 70-mesh screen at end ( $s=3) ; M_{j}=1.27$.

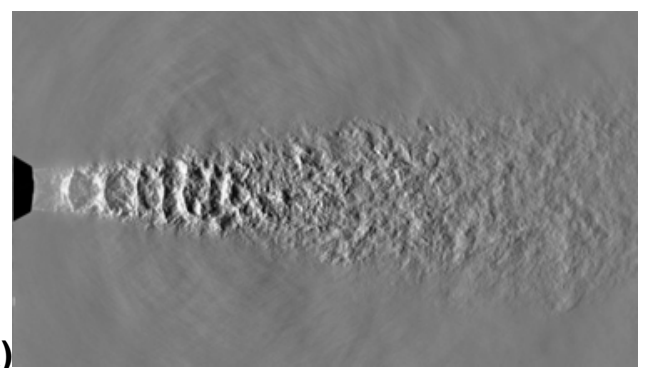

(a)

(b)

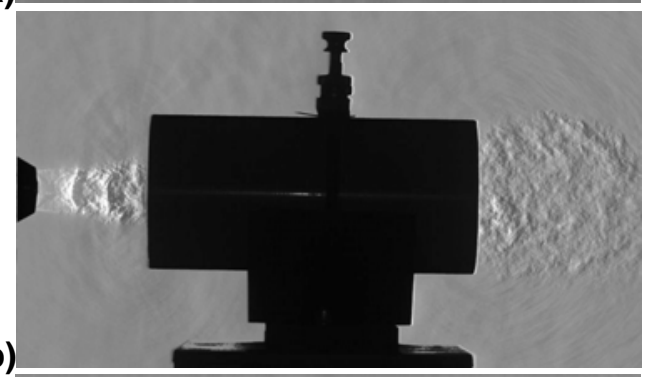

(c)

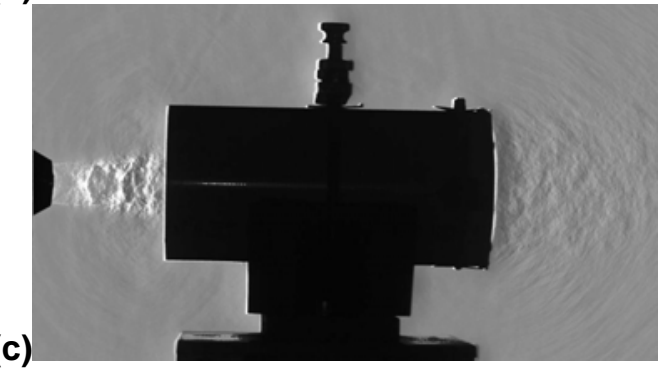

Fig. 14 Schlieren pictures. (a) free tabbed jet, (b) tabbed jet plus duct $(s=3)$, (c) tabbed jet plus duct with 70-mesh screen at end ( $s=3) ; M_{j}=1.38$. 
(a)
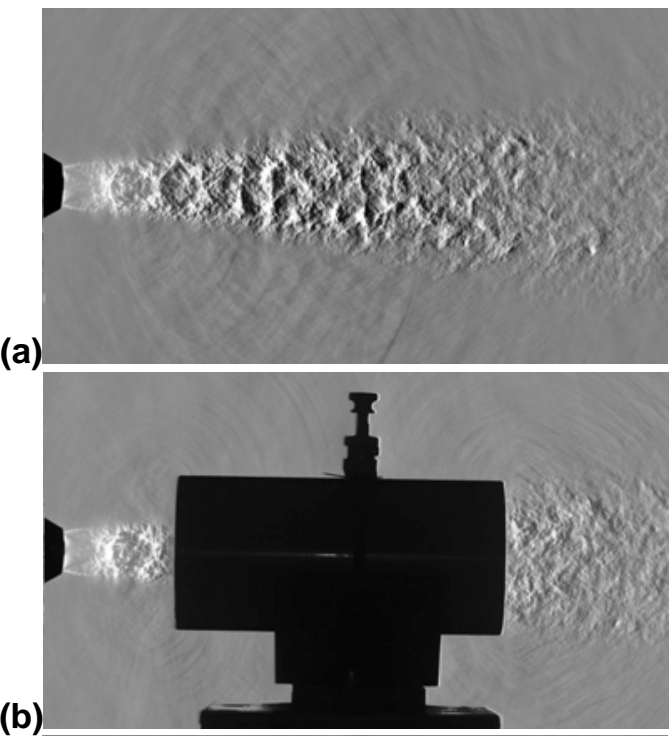

(b)

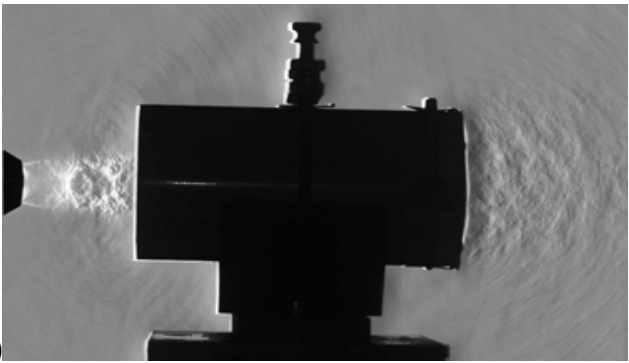

Fig. 15 Schlieren pictures. (a) free tabbed jet, (b) tabbed jet plus duct $(s=3)$, (c) tabbed jet plus duct with 70-mesh screen at end ( $s=3) ; M_{j}=1.60$. 